University of South Carolina

Scholar Commons

$5-6-1987$

\title{
Hard-Sphere Theory for Correlation of Tracer Diffusion of Gases and Liquids in Alkanes
}

\author{
Michael A. Matthews \\ University of South Carolina - Columbia, matthews@cec.sc.edu \\ Aydin Akgerman
}

Follow this and additional works at: https://scholarcommons.sc.edu/eche_facpub

Part of the Chemical Engineering Commons

\section{Publication Info}

Published in The Journal of Chemical Physics, Volume 87, Issue 4, 1987, pages 2285-2291.

(C) The Journal of Chemical Physics 1987, American Institute of Physics.

Matthews, M. A. \& Akgerman, A. (1987). Hard-Sphere Theory for Correlation of Tracer Diffusion of Gases and Liquids in Alkanes. The Journal of Chemical Physics, 87 (4), 2285 - 2291. http://dx.doi.org/10.1063/ 1.453159

This Article is brought to you by the Chemical Engineering, Department of at Scholar Commons. It has been accepted for inclusion in Faculty Publications by an authorized administrator of Scholar Commons. For more information, please contact digres@mailbox.sc.edu. 


\section{A|P| $\begin{aligned} & \text { The Journal of } \\ & \text { Chemical Physics }\end{aligned}$}

Hardsphere theory for correlation of tracer diffusion of gases and liquids in alkanes

Michael A. Matthews and Aydin Akgerman

Citation: The Journal of Chemical Physics 87, 2285 (1987); doi: 10.1063/1.453159

View online: http://dx.doi.org/10.1063/1.453159

View Table of Contents: http://scitation.aip.org/content/aip/journal/jcp/87/4?ver=pdfcov

Published by the AIP Publishing

\section{Articles you may be interested in}

Hard-sphere perturbation theory for a model of liquid $\mathrm{Ga}$

J. Chem. Phys. 129, 024503 (2008); 10.1063/1.2948950

Diffuse interface analysis of crystal nucleation in hard-sphere liquid

J. Chem. Phys. 117, 10121 (2002); 10.1063/1.1519862

Tracer-diffusion in binary colloidal hard-sphere suspensions

J. Chem. Phys. 117, 5908 (2002); 10.1063/1.1501123

Diffusion of xenon in liquid alkanes: Temperature dependence measurements with a new method.

Stokes-Einstein and hard sphere theories

J. Chem. Phys. 92, 625 (1990); 10.1063/1.458413

Inherent structure theory of liquids in the hardsphere limit

J. Chem. Phys. 83, 4767 (1985); 10.1063/1.449840

COMSOL

CONFERENCE

20I4 BOSTON
The Multiphysics Simulation Event of the Year

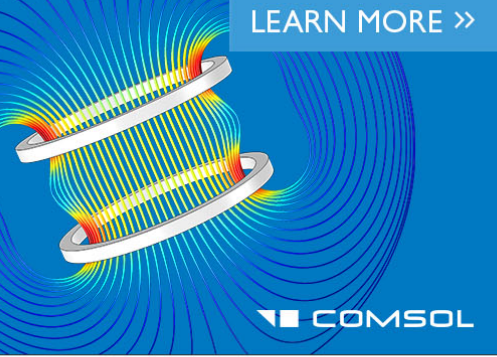




\title{
Hard-sphere theory for correlation of tracer diffusion of gases and liquids in alkanes
}

\author{
Michael A. Matthews and Aydin Akgerman \\ Chemical Engineering Department, Texas A\&M University, College Station, Texas 77843
}

(Received 31 March 1987; accepted 6 May 1987)

\begin{abstract}
The rough hard-sphere (RHS) theory for transport properties has substantially improved understanding of diffusion, viscosity, and thermal conductivity. At present, however, quantitative predictions for polyatomic species are hampered by uncertainties in assigning the hard-sphere diameter and the roughness factor, and by lack of molecular dynamics calculations. Because the qualitative features of the theory have proven correct for a variety of chemical systems, methods have been proposed for using rough hard-sphere theory even when the exact parameters needed to apply the theory are unknown. One such approach is examined for tracer diffusion in alkane solvents for solutes ranging in size from hydrogen to hexadecane. Useful relations are shown which are simple, yet entirely consistent with hard-sphere theory.

The approach circumvents the difficulties in assigning roughness factor, diameter, and molecular dynamics results.
\end{abstract}

\section{INTRODUCTION}

Diffusion coefficients in liquids over extended temperature and pressure ranges are needed for design of mass transfer processes. In addition, the mutual diffusion coefficient is most important in providing information regarding the nature of interactions between unlike molecules. It is thus a valuable quantity in development of a theory on the dynamics of the fluid state. The rough hard-sphere (RHS) approach to diffusion (and other transport properties) considers the dynamics of molecular collisions between rigid, spherical molecules at liquid densities. It is the most successful of theories for diffusion to date. However, most of the applications of hard-sphere theory have been on spherical molecules such as methane and the noble gases. To apply the exact theory, one needs the hard core diameter (which is temperature dependent), the translational/rotational coupling factor (or roughness factor), and the results from molecular dynamics (MD) calculations done with computers. Rigorous application to complicated, flexible polyatomic molecules such as alkanes is still somewhat beyond current capabilities.

Molecular dynamics calculations on hard-sphere systems essentially provides an exact model. MD has been shown to give at least qualitatively correct behavior many times. In light of this qualitatively correct behavior, we examine an approach to predicting tracer diffusion data which bypasses the need for explicitly determining two of the three parameters listed above. The correlation requires only a single hard-core diameter, evaluated at one condition.

\section{THEORY}

Dymond ${ }^{1}$ has recently reviewed the theory and applications of hard-sphere theories to self-diffusion, mutual diffusion, viscosity, and thermal conductivity. The theory for tracer diffusion (mutual diffusion at infinite dilution of the solute) is given below, along with a discussion of the difficulties of rigorous application to polyatomic molecules.
In compact form, the RHS mutual diffusion coefficient is obtained as the product of four terms:

$$
D_{12}=D_{12}^{\mathrm{RHS}}=D_{12}^{o} \cdot \frac{1}{g\left(\sigma_{12}\right)} \cdot C\left(\sigma_{1}, \sigma_{2}, m_{1}, m_{2}, V\right) \cdot A_{12} .
$$

The first term $D_{12}^{0}$ is the mutual diffusion coefficient for a dilute (low density) collection of hard spheres ${ }^{2}$ :

$$
D_{12}^{0}=\frac{3}{8 n \sigma_{12}^{2}}\left[\frac{k T}{2 \pi} \frac{\left(m_{1}+m_{2}\right)}{m_{1} m_{2}}\right]^{1 / 2},
$$

where $n$ is the number density (molecules/volume), $\sigma_{12}=\left(\sigma_{1}+\sigma_{2}\right) / 2$ is the collision (average) diameter of the solute and solvent molecules, and $m_{1}$ and $m_{2}$ are the molecular masses.

At higher densities, the low-density result is scaled in time ${ }^{2}$ by the radial distribution function $g\left(\sigma_{12}\right)$ :

$$
\frac{n D_{12}}{n^{0} D_{12}^{0}}=\frac{1}{g\left(\sigma_{12}\right)}
$$

where $n D_{12}$ is the high-density result and $n^{0} D_{12}^{0}$ is the low density result, Eq. (2).

For an infinitely dilute solution, $g\left(\sigma_{12}\right)$ is usually calculated as follows ${ }^{3,4}$ :

$g\left(\sigma_{12}\right)=\frac{1}{1-\xi}+\frac{3 \xi \sigma_{1}}{(1-\xi)^{2}\left(\sigma_{1}+\sigma_{2}\right)}+\frac{\xi^{2} \sigma_{1}}{2(1-\xi)^{3} \sigma_{2}}$,

where $\xi$ is the packing factor for hard-sphere assemblies. For tracer diffusion $\left(n_{1}=0\right)$

$$
\xi=\frac{\pi n_{2} \sigma_{2}^{3}}{6} .
$$

Equation (4) is based on the Percus-Yevick equation of state for hard spheres. ${ }^{5}$ It may not be valid for values of $\sigma_{1} / \sigma_{2}$ far from unity.

The term $C\left(\sigma_{1}, \sigma_{2}, m_{1}, m_{2}, V\right)$ in Eq. (1) corrects the Enskog theory in the region of liquid densities for back- 
scattering and vortex formation. ${ }^{6-8}$ The correction is obtained by comparing molecular dynamics calculations $\left(D^{\mathrm{HS}}\right)$ for assemblies of smooth hard spheres to the predictions from Enskog theory. The factor $C$ is defined thus:

$$
C\left(\sigma_{1}, \sigma_{2}, m_{1}, m_{2}, V\right)=\frac{D^{\mathrm{HS}}}{\left[D_{12}^{0} / g\left(\sigma_{12}\right)\right]} .
$$

The few available results are usually given as a function of $\sigma_{1} / \sigma_{2}, m_{1} / m_{2}$, and $V / V_{0}$, where $V_{0}$ is the close-packed hard-sphere volume of the solvent:

$$
V_{0}=N \sigma_{2}^{3} / \sqrt{2} \text {. }
$$

Early machine calculations from several sources ${ }^{4,7,10,11}$ have been used extensively in interpretation of diffusion coefficients. However, the accuracy of early molecular dynamics calculations has been questioned by Esteal $e t$ al. based on their more recent calculations. ${ }^{12,13}$ It appears that the early machine calculations may not be sufficiently accurate for quantitative predictions of tracer diffusion.

In collisions between polyatomic molecules, it is possible that kinetic and rotational energy can be exchanged, violating the smoothness assumption of Enskog theory. Chandler ${ }^{14}$ has shown that a roughness factor $A$ [the fourth term in Eq. (1)] should be introduced to account for this. The effect of coupling between rotational and kinetic energy is to reduce the diffusion coefficient, so

$$
0 \leqslant A \leqslant 1 \text {. }
$$

It was originally postulated that, for self-diffusion, $A_{11}$ should be independent of density and only weakly dependent on temperature. A roughness factor for mutual diffusion $A_{12}$ is similarly defined. Baleiko and Davis ${ }^{15}$ calculated $A_{12}$ for rough spheres from geometric considerations and concluded that $0.71<A_{12} \leqslant 1$. Evans et $a l .^{3}$ fitted several sets of diffusion data using values of $A_{12}$ fixed at either 1,0.78, of 0.7 , depending on the constituents of the system. However, other calculations of the roughness factor from experimental data have shown that these limitations do not hold. Values of 0.54 and 0.59 for $A_{12}$ have been found to explain intradiffusion in carbon tetrachloride ${ }^{14}$ and tetramethylsilane. ${ }^{16}$ Bertucci and Flygare ${ }^{17}$ similarly fitted data and found values of $A$ between 0.44 and 0.552 . Esteal and Woolf ${ }^{18}$ have shown that, for certain species, the roughness factor may show temperature or density dependence, or both.

Presently, rigorous predictions from RHS are complicated not only by limited amount of reliable MD data, but also by the uncertainty in assigning unambiguous values for the molecular diameters and roughness factor. According to Esteal and Woolf, ${ }^{19}$ the preferred method for determining $\sigma_{i}(T)$ is from liquid molar volume data along the freezing curve, using the relationship

$$
\sigma_{i}(T) / \mathrm{nm}=0.11611\left(V_{i} / \mathrm{cm}^{3} \mathrm{~mol}^{-1}\right)^{1 / 3} .
$$

However, the high pressure data needed to evaluate $\sigma_{i}$ for an extended temperature range is available for only a few substances. Chandler ${ }^{14}$ determined the diameter for carbon tetrachloride by calculating the logarithmic derivative of selfdiffusivity with density. Again, very high pressure self-diffusion data are seldom available. In an early work, Dymond and Alder ${ }^{20}$ used PVT data to obtain a diameter for methane. Dymond ${ }^{21}$ later demonstrated an alternate method for finding the diameter from self-diffusion data.

For tracer diffusion, one needs two diameters and the roughness factor. If high pressure self-diffusion data or PVT data along the solid-liquid coexistence curve are available for both species, then one may use the methods given above to calculate the diameters. If such data are not available, then one must simultaneously estimate the diameters and the roughness factor. Bertucci and Flygare ${ }^{17}$ examined the derivative of the mutual diffusion coefficient with composition and were able to solve simultaneously for the two component diameters, independently of the roughness factor (which was assumed to be composition independent). Czworniak et $a l .^{22}$ used a similar approach. However, this approach requires taking derivatives of the early MD computer results which have been called into question. ${ }^{12,13}$ In addition, the assumption of composition-independent $\boldsymbol{A}_{12}$ may not be correct. Evans $e$ a $l^{3}$ fixed $A_{12}$ at either $1,0.78$, or 0.7 , depending on the constituents of the system. With self-diffusivities providing the diameter for the solvent, they then used tracer diffusivities to determine a single diameter for the solute.

It is seen that a variety of approaches are used to obtain the roughness factor and the molecular diameters from experimental data. As of yet, a consistent set of data for a wide variety of species is not available. This inconsistency is demonstrated in Table $I$, where the hard-sphere diameter and roughness factor for cyclohexane as determined by various authors are given. Since a $1 \%$ change in diameter may make a difference of several percent in the calculated diffusion coefficient, ${ }^{23}$ it is evident that the present level of uncertainty makes predictions difficult. However, the general RHS theory is qualitatively correct and an alternative analysis is desired which circumvents some of the difficulties of the theory as presented above.

The approach to analysis of tracer diffusion begins with consideration of self-diffusion. It has been found ${ }^{24}$ that the molecular dynamics calculations of the group $C / g(\sigma)$ for self-diffusion can be fitted to a straight line as follows:

$$
\frac{V}{V_{0}} \cdot C\left(\sigma_{1}, m_{1}, V / V_{0}\right) \cdot \frac{1}{g\left(\sigma_{1}\right)}=a\left[\frac{V}{V_{0}}-b\right],
$$

where $V$ is the molar volume of the liquid mixture at the temperature and pressure of the system, $V_{0}$ is defined by Eq. (7), and $a$ is a constant. The constant $b$ has been found empirically to be either $1.384^{24}$ or $1.358^{25}$ based on early MD calculations. The recent molecular dynamics calculations of Esteal and co-workers ${ }^{12}$ can be fit with $b=1.3509$ for selfdiffusion over a wider range of densities $\left(1.5 \leqslant V / V_{0} \leqslant 4.0\right)$.

TABLE I. Hard core diameters and roughness factors for cyclohexane as determined by a number of methods.

\begin{tabular}{ccc}
\hline \hline $\begin{array}{c}\sigma \text { at } 298 \mathrm{~K} \\
(\AA)\end{array}$ & $A_{11}$ & Reference \\
\hline 5.488 & 0.51 & 22 \\
5.54 & 0.70 & 3 \\
5.51 & 0.57 & 23 \\
5.54 & 0.71 & 16 \\
5.64 & 0.70 & 27 \\
\hline
\end{tabular}


A similar functional form is assumed to hold for mutual diffusion, as suggested first by Chen et al. ${ }^{26}$ Substituting Eq. (10) into Eq. (1) and using Eq. (2) to express $D_{12}^{0}$,

$D_{12}=A_{12} \cdot \frac{3}{8 n \sigma_{12}^{2}}\left[\frac{k T}{2 \pi} \frac{\left(m_{1}+m_{2}\right)}{m_{1} m_{2}}\right]^{1 / 2} \cdot \frac{V_{0}}{V}\left[a\left(\frac{V}{V_{0}}-b\right)\right]$.

The product $n V$ in the denominator is Avogadro's number. For a given binary system, $m_{1}$ and $m_{2}$ are constant. The diameters are weakly decreasing functions of temperature. Thus, Eq. (11) can be shortened by collecting constant terms to

$\frac{D_{12}}{\sqrt{T}}=\frac{K}{\sigma_{12}^{2}}\left(\frac{m_{1}+m_{2}}{m_{1} m_{2}}\right)^{1 / 2}\left[a\left(V-b V_{0}\right)\right]=\beta\left(V-V_{D}\right)$.

If one considers data near the saturation curve, $V_{D}$ should depend only on the solvent and represents the solvent molar volume at which diffusion ceases. The constant $\beta$ will depend on both the solute and the solvent masses, diameters, and roughness factors. However, it will be constant for a given solute/solvent pair.

Equation (12) will provide the starting point for analysis of experimental tracer diffusion data. Rough hard sphere theory predicts that $D_{12} / \sqrt{T}$ should form a straight line when plotted vs molar volume. Should this relationship hold, then it would be possible to predict $D_{12}$ for a given solute/solvent pair by using one temperature dependent physical property (the solvent molar volume $V$ ) and two constants, $\beta$ and $V_{D}$.

\section{RESULTS AND DISCUSSION}

The linear relationship given by Eq. (12) has been demonstrated for tracer diffusion of rare gases, methane, and aromatics. ${ }^{26,27}$ Recently, Matthews and Akgerman ${ }^{28-30}$ have reported tracer diffusion for hydrogen, $\mathrm{CO}, \mathrm{CO}_{2}$, and $n$-alkane solutes in a series of $n$-alkane solvents (heptane, dodecane, and hexadecane). The data cover an extensive range of molecular size and mass: $0.0088 \leqslant m_{1} / m_{2} \leqslant 2.26$, $0.32 \leqslant \sigma_{1} / \sigma_{2} \leqslant 1.3$, and $1.4 \leqslant V / V_{0} \leqslant 2.0$. In Figs. $1-3, D_{12} / \sqrt{T}$ is plotted against the solvent molar volume $V$ for diffusion in $n$-alkanes. It is seen that there is indeed a linear relationship which confirms the general predictions of the RHS theory for the wide range of solute masses and sizes.

Values of the slope $\beta$ and intercept $V_{D}$ from Figs. 1-3 are given in Table II. According to theory, the intercept should be strictly a solvent property, but it is observed that $V_{D}$ appears to depend on the nature of the solute molecule. For the alkane solutes, the intercept $V_{D}$ as found from regression analysis is constant within a span of $\pm 1 \%$ of the mean for a given solvent. For the gas solutes, the calculated intercept is consistently about $2 \%$ lower than for the alkanes. The difference in intercepts cannot be ascribed to different "roughness factors" for the gas and alkane molecules. This is contrary to expectations for the idealized hard-sphere systems, for which one would expect a single limiting value of the solvent molar volume. However, this result is still physically reasonable. Viewed from a free-volume standpoint,

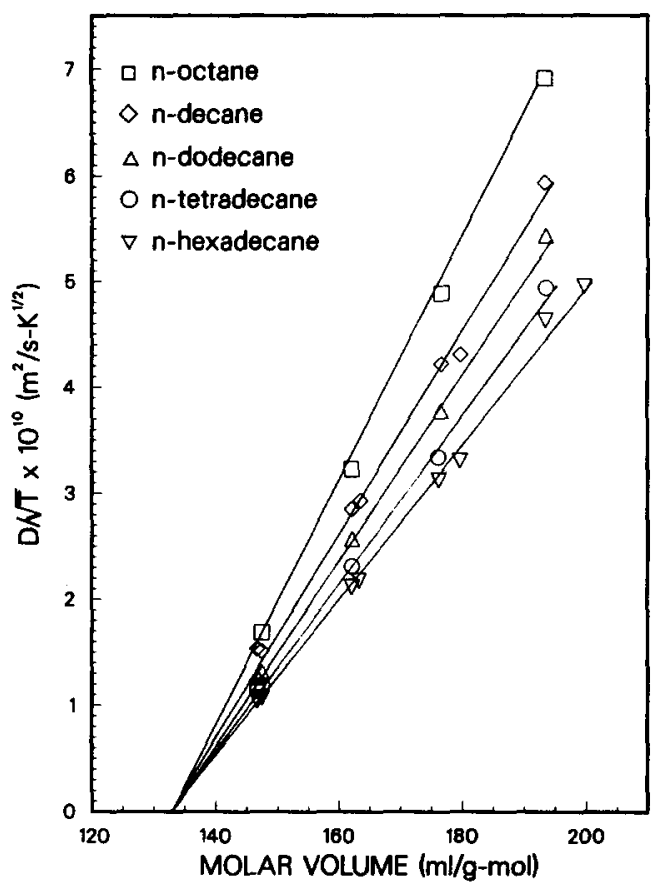

FIG. 1. Diffusivities of alkanes in $n$-heptane, according to Eq. (12).

this implies that the smaller gas solutes are more mobile within the solvent free space than the alkane solutes.

The limiting solvent volume should be near the pure solvent freezing volume, which is also shown in Table II. As expected, $V_{D}$ is very close to $V_{t p}$, i.e., the diffusion coefficient goes to zero near the point where the solvent is making the phase change from liquid to solid. However, exact correspondence of $V_{D}$ to $V_{t p}$ is not expected because the linear extrapolation to obtain $V_{D}$ is made using data where $V / V_{0}$ is

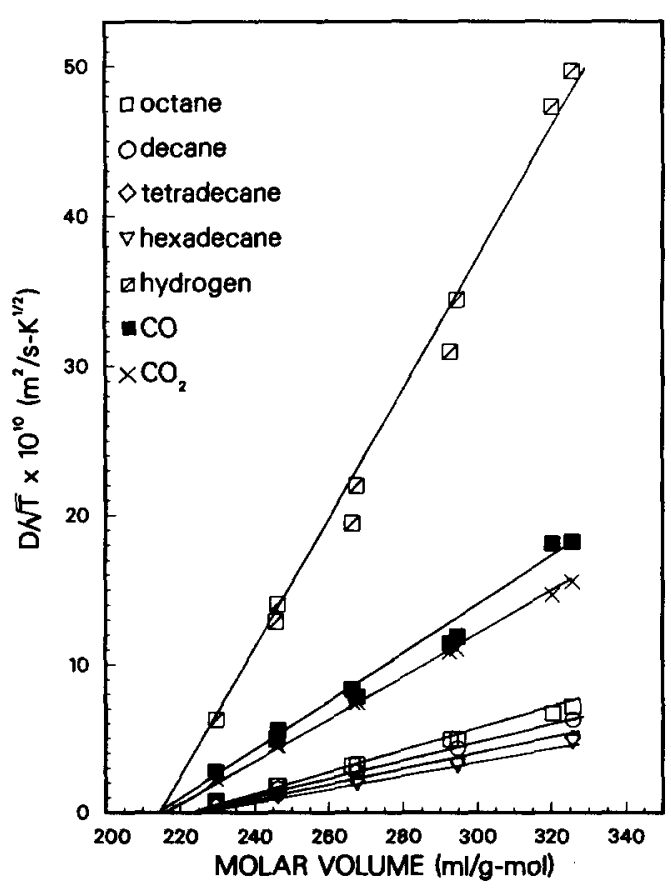

FIG. 2. Diffusivities of gases and alkanes in $\boldsymbol{n}$-dodecane, according to Eq (12). 


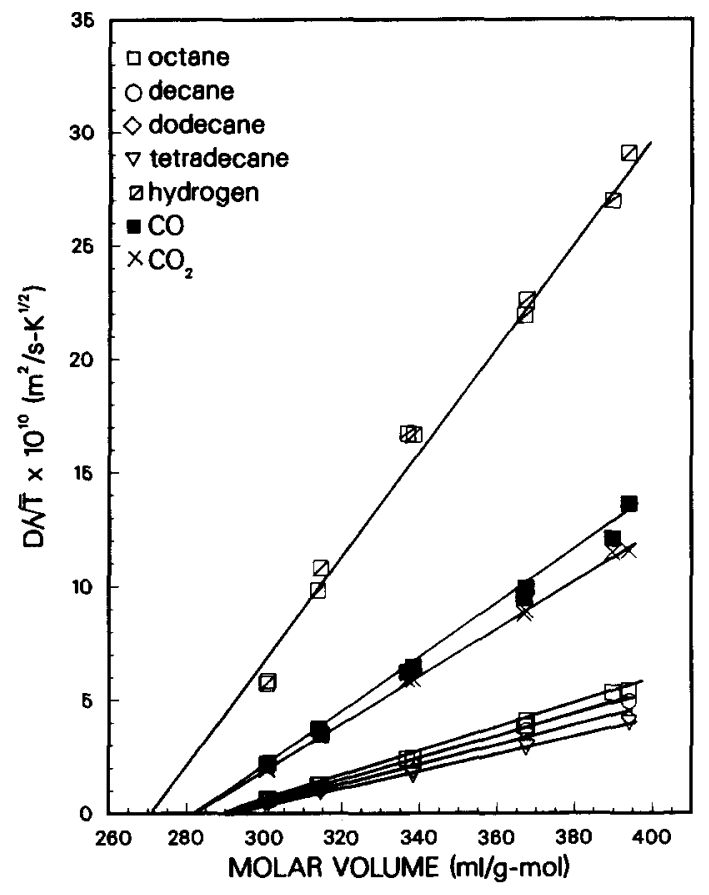

FIG. 3. Diffusivities of gases and alkanes in $n$-hexadecane, according to Eq. (12).

greater than 1.4. At higher densities, RHS theory is known to break down. In addition, because the limiting volume depends on the nature of the diffusing solute, one cannot fix the value of $V_{D}$ to the pure component freezing point, which is an equilibrium property.

TABLE II. Coefficients for Eq. (11).

\begin{tabular}{|c|c|c|}
\hline Solute & $\beta$ & $V_{D}$ \\
\hline \multicolumn{3}{|c|}{ Solvent: $n$-heptane. $V_{c}=432, V_{\mathrm{tp}}=129$} \\
\hline Octane & 0.01143 & 133.3 \\
\hline Decane & 0.009687 & 132.6 \\
\hline Dodecane & 0.008799 & 132.5 \\
\hline Tetradecane & 0.00802 & 133.0 \\
\hline Hexadecane & 0.007511 & 133.7 \\
\hline Hydrogen & 0.06980 & 127.6 \\
\hline $\mathrm{CO}$ & 0.02591 & 129.3 \\
\hline \multicolumn{3}{|c|}{ Solvent: $n$-dodecane. $V_{\mathrm{c}}=713, V_{\mathrm{tp}}=221$} \\
\hline Octane & 0.006647 & 219.0 \\
\hline Decane & 0.005813 & 219.3 \\
\hline Tetradecane & 0.004729 & 221.2 \\
\hline Hexadecane & 0.004482 & 221.9 \\
\hline Hydrogen & 0.04491 & 218.0 \\
\hline $\mathrm{CO}$ & 0.01618 & 215.4 \\
\hline $\mathrm{CO}_{2}$ & 0.1375 & 213.3 \\
\hline \multicolumn{3}{|c|}{ Solvent: $n$-hexadecane. $V_{c}=930,{ }^{a} V_{\mathrm{tp}}=292^{\mathrm{b}}$} \\
\hline Octane & 0.005157 & 289.5 \\
\hline Decane & 0.004750 & 291.3 \\
\hline Dodecane & 0.003975 & 288.7 \\
\hline Tetradecane & 0.003773 & 290.3 \\
\hline Hydrogen & 0.029314 & 280.2 \\
\hline $\mathrm{CO}$ & 0.01167 & 282.9 \\
\hline $\mathrm{CO}_{2}$ & 0.01041 & 281.7 \\
\hline
\end{tabular}

${ }^{\mathrm{a}} V_{c}=$ critical volume.

${ }^{\mathrm{b}} V_{\mathrm{tp}}=$ liquid volume at triple point $\left(\mathrm{cm}^{3} / \mathrm{mol}\right)$.
Although the limiting solvent volume is not a constant, it is instructive to calculate the hard sphere diameter from $V_{D}$ :

$$
V_{D}=b V_{0}=b N \sigma_{2}^{3} / \sqrt{2},
$$

where $N$ is Avogadro's number. The constant $b$ for self-diffusion has been found to be $1.384^{24}, 1.358^{25}$, or 1.3509 from linear regression of the data of Esteal et al. ${ }^{12}$ Using $b=1.3509$ and the average $V_{D}$ for the alkane solutes gives $\sigma=6.13,7.26$, and $7.95 \AA$ for $n$-heptane, $n$-dodecane, and $n$ hexadecane, respectively. To compare with the calculated diameters, we use the group contribution method of Bondi ${ }^{31}$ to obtain the van der Waals volumes of the molecules, from which we get the diameter of a sphere of equal volume. The van der Waals diameters are given in Table III. It it seen that there is good agreement, but the differences reflect the inability of the molecular models to accurately describe both transport and equilibrium properties.

In light of these observations, $V_{D}$ must be regarded as a fictitious quantity with a small but nonneglibible dependence on the nature of the solute. However, this does not eliminate the potential usefulness of this analysis as a predictive approach.

A method for predicting $V_{D}$ is necessary to use Eq. (12) as a predictive tool. The solvent critical volumes are also given in Table II and it is found that for alkane solutes, the solvent property $V_{D}=0.308 V_{c}$ on the average. For the gaseous solutes, $V_{D}=0.302 V_{c}$. Thus it is seen that for $n$-alkane solvents the parameter $V_{D}$ could be estimated simply from the critical volume of the solvent.

The more difficult task is to explain and predict the slope $\beta$. Although the relationship given by Eq. (12) has been observed many times, no extensive systematic investigation of $\beta$ has been attempted. The present work provides enough information to allow development of some predictive methods to be used in $n$-alkane systems.

In light of the lack of reliable MD data, it is necessary to use the experimental data to establish ways to determine the slope $\beta$ empirically. This constant contains all the information on the interactions between unlike particles 1 and 2 . RHS theory dictates that $\beta$ is a function of the following variables: solute and solvent masses, diameters, and the roughness factor $A_{12}$. In seeking a representation for the slope $\beta$, several requirements were enforced. First, we desired an equation with as few parameters as possible, with the $F$ test for each parameter indicating a high level of statistical significance. It was anticipated that gas and alkane dif-

TABLE III. Core diameters from procedure of Bondi.

\begin{tabular}{llll}
\hline \hline & $\sigma(\AA)$ & & $\sigma(\AA)$ \\
\hline $\mathrm{H}_{2}$ & 2.572 & $\mathrm{C}_{7}$ & 6.29 \\
$\mathrm{CO}$ & 3.718 & $\mathrm{C}_{8}$ & 6.552 \\
$\mathrm{CO}_{2}$ & 3.968 & $\mathrm{C}_{9}$ & 6.795 \\
$\mathrm{CH}_{4}$ & 3.785 & $\mathrm{C}_{10}$ & 7.022 \\
$\mathrm{Ar}$ & 3.76 & $\mathrm{C}_{12}$ & 7.436 \\
$\mathrm{Kr}$ & 4.04 & $\mathrm{C}_{14}$ & 7.808 \\
$\mathrm{Xe}$ & 4.32 & $\mathrm{C}_{16}$ & 8.148 \\
\hline
\end{tabular}


fusivities might not be correlated in a single equation due to the large differences in molecular weight, size, and shape. However, we desired that the same functional form be used to represent both gas and liquid solutes. Also, we desired that only the variables suggested by RHS theory be employed in the correlation; thus arbitrary use of viscosity or an activation energy was not allowed.

Several representations were found which adequately correlated $\beta$ for either the gas or liquid solutes. However, only one representation satisfied all the criteria. $\beta$ for a given solute/solvent pair can be represented within experimental uncertainty by use of just two variables, namely, the molecular weight $M_{1}$ and the solute/solvent size ratio $\sigma_{1} / \sigma_{2}$. Different constants are required to represent both gas and liquid solutes. The functional form for the slope $\beta$ is given in $\mathrm{Eq}$. (14):

$$
\beta=a M_{1}^{b}\left(\frac{\sigma_{1}}{\sigma_{2}}\right)^{c}
$$

For alkane solutes, $a=15.8 \pm 1.2, b=-1.56 \pm 0.03$, and $c=2.99 \pm 0.06$, where the uncertainties are one standard deviation. For dissolved gas solutes, $a=1.65 \pm 1.11$, $B=-0.76 \pm 0.02$, and $c=3.02 \pm 0.08$.

Therefore the high temperature behavior of the diffusion coefficient as predicted by hard-sphere theory can be summarized for the systems investigated in this study as:

$$
\frac{10^{9} D_{12}}{\sqrt{T}}=a M_{1}^{b}\left(\frac{\sigma_{1}}{\sigma_{2}}\right)^{3}\left(V-V_{D}\right),
$$

where, for $n$-alkane solutes

$$
V_{D}=0.308 V_{c}, \quad b=-1.56, \quad a=15.8,
$$

and for dissolved gas solutes

$$
V_{D}=0.302 V_{c}, \quad b=-0.76, \quad a=1.65 \text {. }
$$

In these equations, $D_{12}$ is in $\mathrm{m}^{2} / \mathrm{s}, T$ is in kelvins, and the molar volumes are in $10^{-6} \mathrm{~m}^{3} / \mathrm{mol}$. For lack of a consistent set of hard-sphere diameters $\sigma_{1}$ and $\sigma_{2}$ were computed by using the group contribution methods of Bondi ${ }^{31}$ as described earlier. This method has the advantages that it is applicable to a variety of compounds in addition to alkanes, and that the volumes are all based on a consistent experimental technique, namely use of $x$-ray diffraction in crystals to obtain contact distances. This relieves the need for high pressure PVT or self-diffusion data to calculate the diameters. Since the diameters appear in Eq. (14) as a ratio, the exact magnitude of the diameters used is unimportant as long as the ratio is correct.

It was interesting to see that, although the exponents of Eq. (15) were determined from regression analysis and were not fixed, the ratio $\left(\sigma_{1} / \sigma_{2}\right)$ is raised to the third power for both gas and alkane solutes. Thus the diffusion coefficient is controlled by the relative volumes occupied by the molecules. This is a physically pleasing result. One could of course replace the hard-core diameters with the hard-core van der Waals volumes, adjusting the constant $a$ accordingly.

Equation (15) was developed from diffusivities of $n$ alkanes. The average absolute percent deviation from the experimental data is $5.5 \%$, with a maximum of about $20 \%$.
To test the generality of Eq. (15), data from the literature were sought. There were only two sources of data for alkane/ alkane systems covering temperatures above $323 \mathrm{~K}$. Alizadeh and Wakeham ${ }^{32}$ give mutual diffusion coefficients in binary mixtures of hexane, heptane, and octane up to $343 \mathrm{~K}$. We used only their data at infinite dilution. Self-diffusivities are reported by Ertl and Dullien ${ }^{33}$ for solvents from heptane to hexadecane at temperatures from 298 to $443 \mathrm{~K}$. The core sizes used are given in Table III. A plot of measured values vs the predictions from Eqs. (15) and (16) is given in Fig. 4 (Fig. 4 does not include any of our data). There is excellent agreement, with an average absolute percent deviation of $7.9 \%$ and a maximum of $31 \%$. The largest percentage errors occur at low temperatures. Since the absolute value of the diffusion coefficient is quite low at these temperatures, the magnitudes of the error can be small and still give a large percentage error.

It was also desired to test Eq. (15) for diffusivities of other gases in $n$-alkanes. Unfortunately there are even fewer data for gases at high temperatures than there are for liquid solutes. The data on diffusivities of methane, argon, krypton, and xenon in alkane solvents given by Chen et $a l^{26}$ was used for comparison (Fig. 5). Also on this figure are the experimental values for $\mathrm{CO}, \mathrm{H}_{2}$, and $\mathrm{CO}_{2}$ measured in this study. For polyatomic gases, the average absolute deviation was $6.6 \%$, with the largest deviation being $28 \%$. For monatomic gases we determined that the value of the intercept value to be used was $0.299 V_{c}$. With this value for $V_{D}$, Eq. (15) was in error by an average of $22 \%$, with all the predictions being low. This is not surprising if one recalls that monatomic solutes are theoretically perfectly smooth, while polyatomic molecules are rough. Since the correlation is based on data with polyatomic species, it incorporates a roughness factor

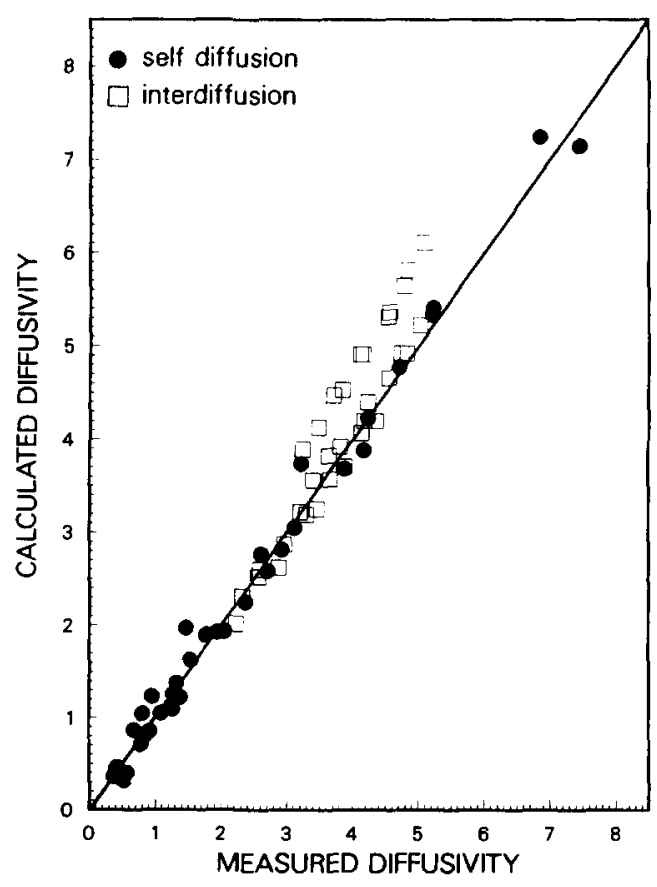

FIG. 4. Comparison of predictions from Eqs. (15) and (16) to tracer diffusivities of $n$-alkanes ${ }^{32} \square$ and self-diffusivities ${ }^{33}$. 


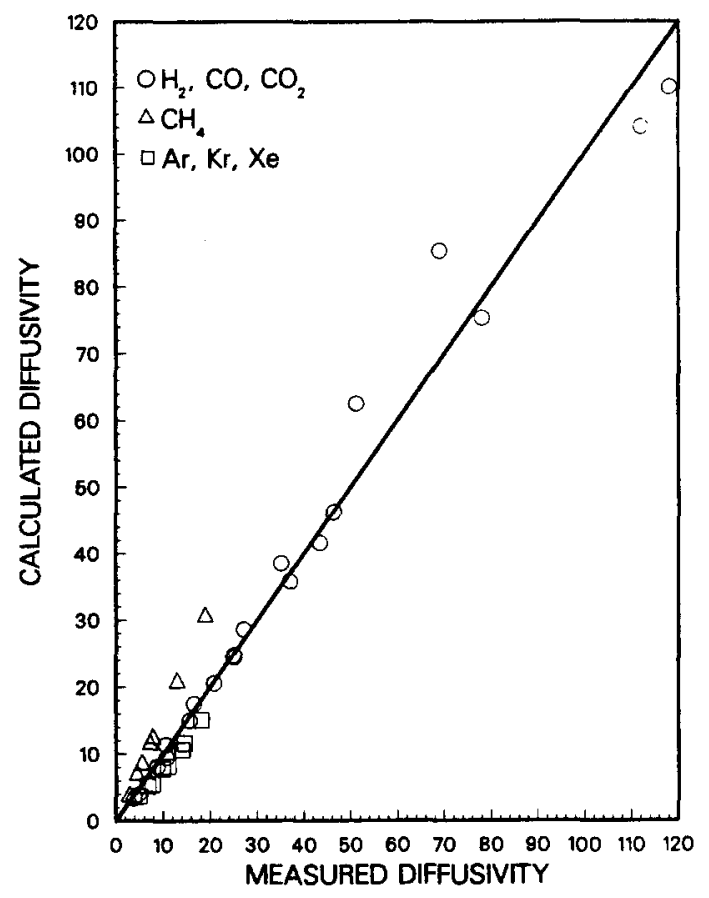

FIG. 5. Comparison of predictions from Eq. (15) and (17) to tracer diffusivities of noble gases $\square$ and methane ${ }^{3} \triangle$. Also shown are the diffusivities of hydrogen, $\mathrm{CO}$, and $\mathrm{CO}_{2}$, upon which the correlation was based $\mathrm{O}$.

less than unity, and it would be expected that monatomic solutes would have a higher diffusivity. The performance of Eq. (15) for noble gases suggests that the ratio of roughness factors between monatomic and polyatomic gases is about 1.22. Previously, Evans et $a l^{3}{ }^{3}$ suggested a roughness factor $A=0.78$ for monatomic solute/polyatomic solvent systems, and $\mathbf{0 . 7}$ for polyatomic solute/polyatomic solvent systems. The ratio of 0.78 to 0.7 is 1.11 , somewhat lower than the present observation.

For methane the experimental diffusivities are unexpectedly lower than the predicted values. Previous studies have claimed that methane, although polyatomic, acts as a model smooth hard-sphere molecule. ${ }^{19}$ One would then expect the methane diffusivities to lie above the predicted values, as did the diffusivities for the noble gases. Figure 6 dem-

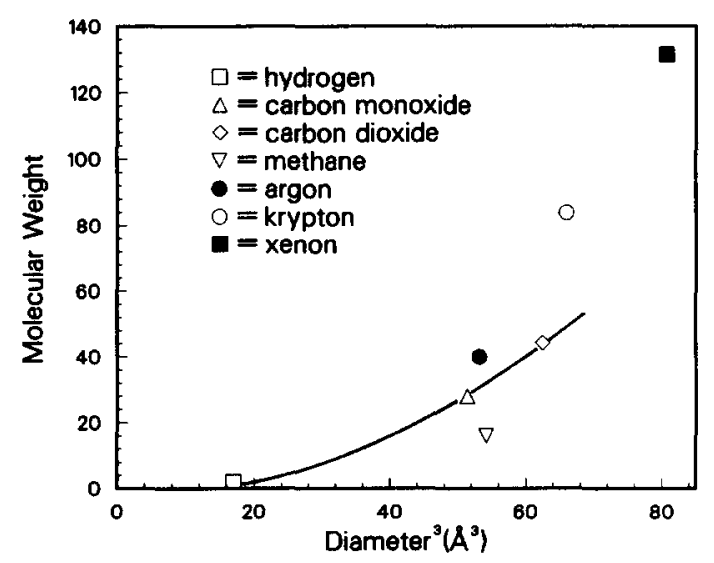

FIG. 6. Mass/volume relationship for $\mathrm{H}_{2} \square, \mathrm{CO} \triangle, \mathrm{CO}_{2} \diamond, \mathrm{Ar} \bullet, \mathrm{KrO}, \mathrm{Xe}$ $\square$, and $\mathrm{CH}_{4} \nabla$. The diameters are calculated from the van der Waals volumes according to Bondi. ${ }^{31}$ onstrates a possible explanation for this inconsistency. This figure shows the molecular weight as a function of the van der Waals diameter cubed, i.e., the molecular mass/volume relationship. A curve is drawn through the points for hydrogen, $\mathrm{CO}$, and $\mathrm{CO}_{2}$. It is seen that methane, while being lighter than either $\mathrm{CO}$ or $\mathrm{CO}_{2}$, has about the same volume. Its motion is thus more hindered by backscattering than $\mathrm{CO}$ or $\mathrm{CO}_{2}$. Hydrogen, while very light, is also very small, so its low mass is compensated for by its low volume. It is also seen that the noble gases have the highest mass to volume ratios, and thus move through the solvent with less backscattering than the polyatomic gases. This gives another explanation for the higher diffusivities of the noble gases, in addition to the argument based on smoothness.

\section{CONCLUSIONS}

It is concluded that the approach to RHS theory summarized by Eq. (12) is a promising method of correlating and predicting diffusion coefficients in chemically similar systems. Only two parameters are needed, a limiting volume $V_{D}$ which corresponds closely but not precisely to the liquid triple point volume, and a slope $\beta$ which expresses the interactions between solute and solvent molecules in terms of their respective hard-core diameters and the solute molecular weight. It is shown experimentally that the limiting solvent molar volume at which diffusivity ceases is not a constant, but is a function of the solute molecule also. The correlation for tracer diffusion of alkane solute applies also to self-diffusion in alkanes over an extended temperature range. The correlation for tracer diffusion of gases does not perform well for diffusion of noble gases and methane. This is ascribed to the low mass/volume ratio for methane and the high mass/volume ratio for the noble gases. Further experiments may be used to investigate this approach, with the goal being a generalized correlation for diffusion coefficients applicable across a wide range of temperatures and pressures. Data are particularly needed for tracer diffusion at very high pressures and across an appreciable temperature range, to determine the dependency of $V_{D}$ on pressure.

\section{ACKNOWLEDGMENT}

This work was supported by the US Department of Energy under contract No. DE AC22 84PC 70032.

'J. H. Dymond, Chem. Soc. Rev. 14, 317 (1985).

${ }^{2} \mathrm{~S}$. Chapman and T. G. Cowling, The Mathematical Theory of NonUniform Gases, 3rd. ed. (Cambridge University, London, 1970).

${ }^{3}$ D. F. Evans, T. Tominaga, and H. T. Davis, J. Chem. Phys. 74, 1298 (1981).

${ }^{4}$ B. J. Alder, W. E. Alley, and J. H. Dymond, J. Chem. Phys. 61, 1415 (1974).

${ }^{5}$ J. L. Lebowitz, Phys. Rev. 133, A895 (1964).

${ }^{6} J$. H. Dymond, J. Chem. Soc. Faraday Trans. 2, 68, 1789 (1972).

${ }^{7}$ B. J. Alder, D. M. Gass, and T. E. Wainwright, J. Chem. Phys. 53, 3813 (1970).

${ }^{8}$ B. J. Alder and T. E. Wainwright, Phys. Rev. A. 1, 18 (1970).

${ }^{9} \mathrm{~S}$. -H. Chen, Ph. D. dissertation, University of Minnesota, Minneapolis, Minnesota, 1980.

${ }^{10}$ P. T. Herman and B. J. Alder, J. Chem. Phys. 56, 987 (1972).

${ }^{11}$ W. W. Shelton, Ph. D. dissertation, University of Minnesota, Minneapolis, 1981.

${ }^{12}$ A. J. Esteal, L. A. Woolf, and D. L. Jolly, Physica A 121, 286 (1983). 
${ }^{13}$ A. J. Esteal and L. A. Woolf, Chem. Phys. 88, 101 (1984).

${ }^{14}$ D. Chandler, J. Chem. Phys. 62, 1358 (1975).

${ }^{15}$ M. O. Baleiko and H. T. Davis, J. Phys. Chem. 78, 1564 (1974).

${ }^{16}$ H. J. Parkhurst and J. Jonas, J. Chem. Phys. 63, 2698 (1975).

${ }^{17}$ S. J. Bertucci and W. H. Flygare, J. Chem. Phys. 63, 1 (1975).

${ }^{18}$ A. J. Esteal and L. A. Woolf, Physica B 124, 182 (1984).

${ }^{19}$ A. J. Esteal and L. A. Woolf, Physica B 124, 173 (1984)

${ }^{20}$ J. H. Dymond and B. J. Adler, J. Chem. Phys. 48, 343 (1968).

${ }^{21}$ J. H. Dymond, Physica 75, 100 (1974).

${ }^{22}$ K. J. Czworniak, H. C. Andersen, and R. Pecora, Chem Phys. 11, 451 (1975).

${ }^{23}$ J. H. Dymond, J. Phys. Chem. 85, 3291 (1981).
${ }^{24}$ J. H. Dymond, J. Chem. Phys. 60, 969 (1974).

${ }^{25}$ H. J. V. Tyrrell and K. R. Harris, Diffusion in Liquids (Butterworths, London, 1984).

${ }^{26}$ S. -H. Chen, H. T. Davis, and D. F. Evans, J. Chem. Phys. 77, 2540 (1982).

${ }^{27}$ C. K. J. Sun and S. -H. Chen, AIChE J. 31, 1510 (1985).

${ }^{28}$ M. A. Matthews and A. Akgerman, AIChE J. 33, 881 (1987).

${ }^{29}$ M. A. Matthews and A. Akgerman, J. Chem. Eng. Data (in press).

${ }^{30}$ M. A. Matthews and A. Akgerman, J. Chem. Eng. Data (in press).

${ }^{31}$ A. Bondi, J. Phys. Chem. 68, 441 (1964).

${ }^{32}$ A. Alizadeh and W. A. Wakeham, Int. J. Thermophys. 3, 307 (1982).

${ }^{33}$ H. Ertl and F. A. L. Dullien, AIChE J. 19, 1215 (1973). 